\title{
Accumulation of an unusual trichothecene shunt metabolite in liquid culture of Fusarium graminearum with methionine as the sole nitrogen source
}

\section{Keywords}

deacetylation; Fusarium graminearum; oxygenation step; shunt intermediate; trichothecene; unusual metabolite

Fusarium graminearum produces type B trichothecenes in liquid culture as the sole trichothecene products. When methionine was used as a nitrogen source, a considerable amount of 3-deacetylcalonectrin (3-deCAL), devoid of a C-8 ketone, accumulated in the synthetic medium as one of the major end products. This indicates that a wild-type $F$. graminearum type $B$ trichothecene producer could accumulate a substantial amount of a type A trichothecene, depending on culture conditions.

Type B trichothecenes are the major mycotoxins that cause toxicity to humans and animals through ingestion of contaminated agricultural products ${ }^{1)}$. For human consumption, a provisional maximum tolerable daily intake (PMTDI) was set for deoxynivalenol (DON) and its acetylated derivatives by the Joint Food and Agriculture Organization (FAO)/World Health Organization (WHO) Expert Committee on Food Additives (JECFA). Following the determination of a PMTDI for DON, many government jurisdictions have set limits for a group of trichothecenes and have imposed an inspection program for cereal grains ${ }^{2}$. In our studies of the effect of different nitrogen sources on the amount of trichothecenes produced by F. graminearum, we discovered an unidentified trichothecene. This letter reports identification of a type $A$ trichothecene as one of the major end products of wild-type F. graminearum, which was identified previously only as the product of the Fgtri1- mutant (a mutant with a disrupted copy of FgTri1, encoding calonectrin C-7/C-8 hydroxylase) ${ }^{3)}$, not the wild-type strain.

F. graminearum JCM 9873, a 15-acetyldeoxynivalenol (15-ADON) producer ${ }^{4}$, was cultured in $30 \mathrm{~mL}$ synthetic medium containing methionine as the sole nitrogen source (Met medium; Supplementary Table 1). The ethyl acetate extract of the culture was analyzed by thin layer chromatography (TLC), as described previously ${ }^{5}$. A blue spot $\left(\mathbf{1} ; R_{\mathrm{f}}=0.50\right)$ of trichothecene with an $R_{f}$ value slightly larger than that of 15-ADON $\left(R_{\mathrm{f}}=0.45\right)$ was detected in addition to 15-ADON. The use of other amino acids as a nitrogen source did not stimulate production of $\mathbf{1}$ in cultures with 2 weeks of incubation (data not shown). Time course analysis of the culture extract revealed that the timing of 15ADON accumulation preceded that of $\mathbf{1}$ in the Met medium (Fig. 1A).

To characterize the structure of $\mathbf{1}$, the relevant area of the silica gel layer of the TLC plate was scraped off and the compound eluted with ethyl acetate as described previously ${ }^{6)}$. When $\mathbf{1}$ was incubated with TRI1017), trichothecene 3-O-acetyltransferase, or TRI3 ${ }^{8)}$, trichothecene 15-O-acetyltransferase, in the presence of $1 \mathrm{mM}$ acetyl CoA, acetyl conjugation of $\mathbf{1}$ was only observed by incubation with TRI101 (Fig. 1B). This suggests that an $\mathrm{O}$-acetyl group is not attached to $\mathrm{C}-3$ of 1. For further characterization, the compound was analyzed by liquid chromatography-tandem mass spectrometry (LC-MS/MS) using the $A B$ SCIEX Triple TOF4600 system (AB Sciex, Framingham, MA) as previously described ${ }^{3)}$. Samples were separated using the Eksigent ekspert ${ }^{\mathrm{TM}}$ ultraLC $100-\mathrm{XL}$ system (AB Sciex) with a $\mathrm{C} 18$ reverse phase column (PEGASIL ODS SP1003; $2 \varphi \times 100 \mathrm{~mm}$, Senshu Scientific Co., Ltd., Tokyo, Japan). A flow rate of $0.3 \mathrm{~mL} / \mathrm{min}$ was employed with a linear gradient from 10 to $95 \%$ acetonitrile in $0.1 \%$ ammonium formate over $5 \mathrm{~min}$. Extracted ion chromatograms of $\mathrm{m} / \mathrm{z} 309.170 \pm 0.025$ and $326.196 \pm$ 0.025 , corresponding to [3-deacetylcalonectrin (3$\mathrm{deCAL})+\mathrm{H}]^{+}\left(\mathrm{m} / \mathrm{z}\right.$ 309.1697) and $\left[3-\mathrm{deCAL}+\mathrm{NH}_{4}\right]^{+}$ $(\mathrm{m} / \mathrm{z}$ 326.1962), showed peaks at $3.672 \mathrm{~min}$ (3.579$3.737 \mathrm{~min}$ ) and $3.644 \mathrm{~min}(3.576-3.729 \mathrm{~min})$, respectively (Fig. 1C). From the tandem mass spectra, 1 was unambiguously identified as 3-deCAL because the fragmentation patterns were superimposable to those of the 3-deCAL standard (Supplementary Fig. S1).

The finding that metabolism of methionine by $F$. graminearum is associated with accumulation of a considerable amount of a trichothecene shunt intermediate, 3-deCAL, suggests that an enzyme responsible for the A-ring modification, FgTRI19), is partially inhibited by methionine or, more likely, by some unidentified metabolites of the fungus under the culture condition used. Alternatively, a deacetylase responsible for C-3 deacetylation of CAL is activated under the culture condition used, resulting in competition with FgTRI1 for the CAL substrate and in an unusually enhanced accumulation of 3-deCAL. To our knowledge, this is the first report on the detection of a substantial amount of 3deCAL from the culture of wild-type F. graminearum. 
(A)

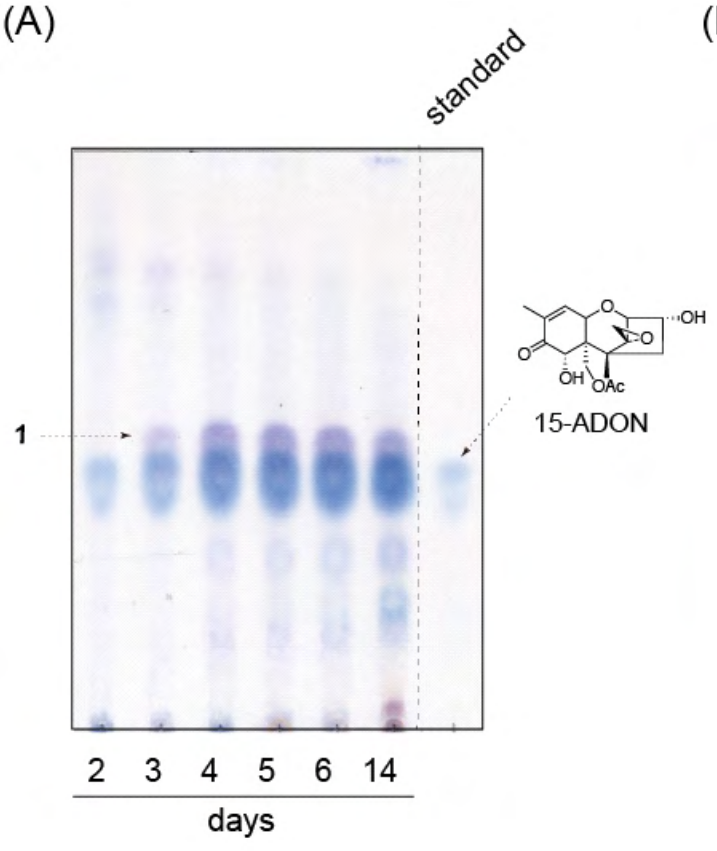

(B)

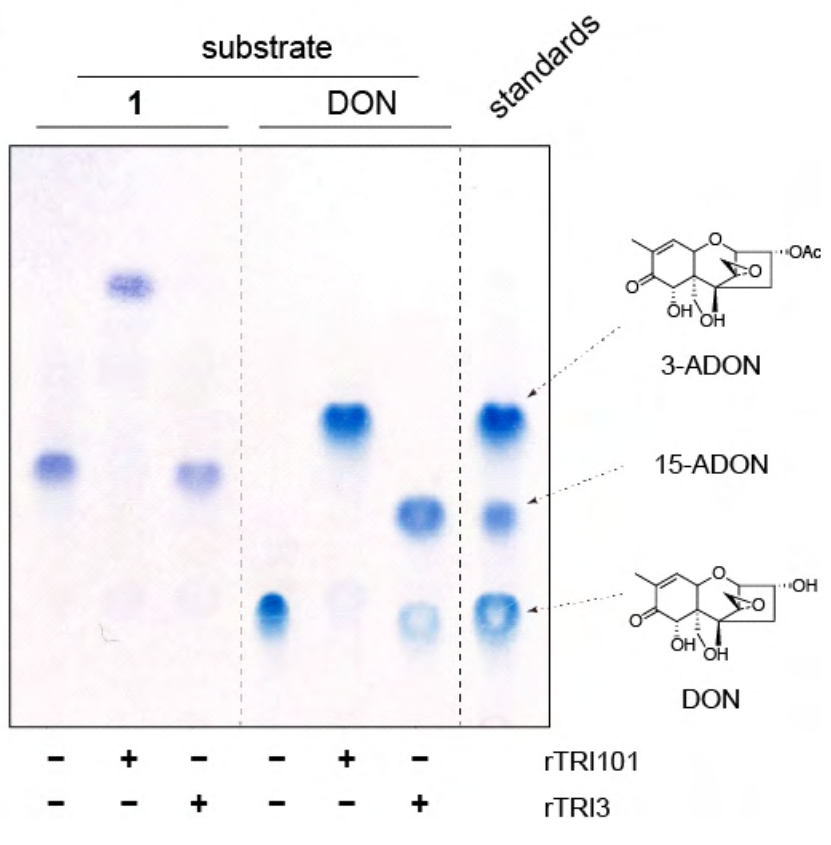

(C)

Product ion spectrum of $\mathrm{m} / \mathrm{z} 309.2$

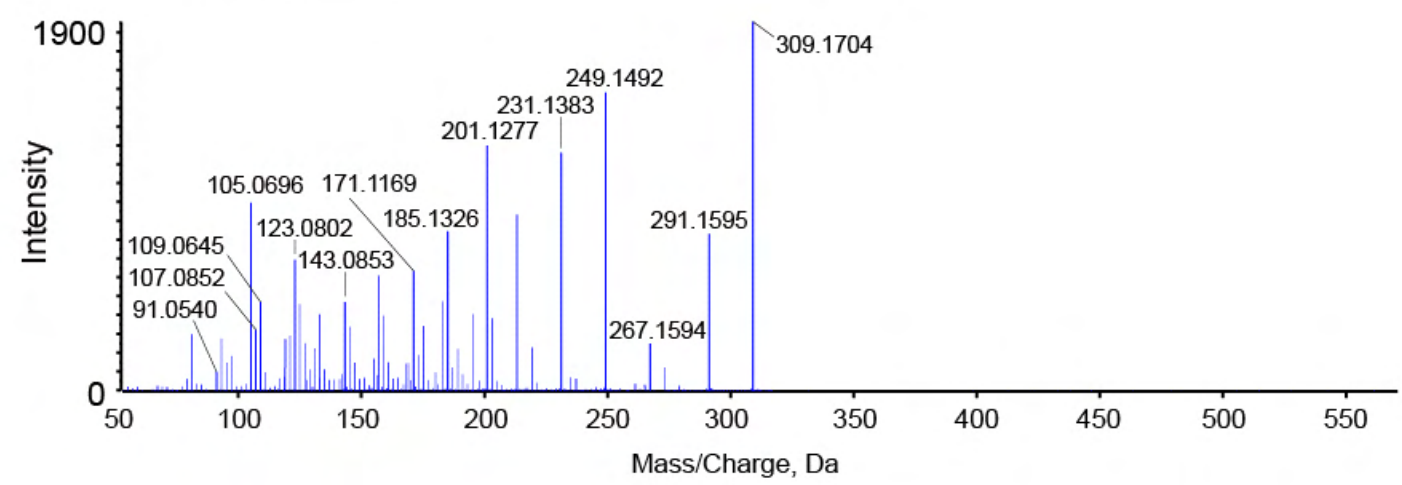

Product ion spectrum of $m / z 326.2$

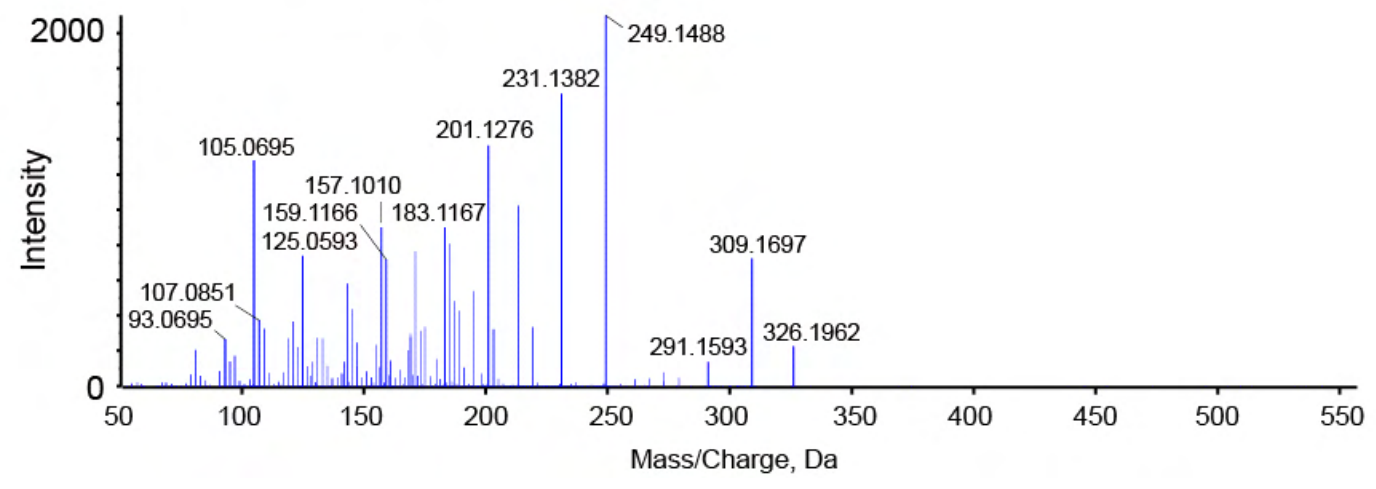

Fig. 1 Characterization of an unusual end product of $F$. graminearum that accumulated in a methionine medium (Met medium). (A) Time course of changes of 15-ADON and $\mathbf{1}$. The metabolites were extracted from the culture with ethyl acetate. The TLC was developed and visualized as described previously). (B) Acetyltransferase assays with purified $\mathbf{1}$. Recombinant TRI3 and TRI101 (rTRI3 and rTRI101) were prepared and acetyltransferase assays were performed as described ${ }^{11), 12)}$. DON was used as a reference substrate to demonstrate activity of the recombinant enzymes. (C) LC-MS/MS analyses of $\mathbf{1}$. The tandem mass spectra of precursor ions were detected in the positive ion mode. $\mathrm{m} / \mathrm{z} 309.2$ and $\mathrm{m} / \mathrm{z} 326.2$ correspond to [3-deCAL $+\mathrm{H}]^{+}(\mathrm{m} / \mathrm{z}$ 309.1697) and [3-deCAL $\left.+\mathrm{NH}_{4}\right]^{+}(m / z$ 326.1962), respectively. 
Although methionine may not occur abundantly in cereal grain spikeletes where the fungus causes infection and produces the mycotoxins, the present result raises the possibility of accumulation of a significant amount of unexpected mycotoxin shunts under some unusual nutritional and environmental conditions. Indeed, although not present in a large amount, a trace of 3-deCAL was detected in all four Japanese wheat samples analyzed by LC-MS/MS ${ }^{10)}$. This suggests the feasibility of this shunt intermediate being present in cereal samples. Thus, in addition to the usual screening program for a regulated mycotoxin such as DON and its derivatives, an occasional survey for trichothecene intermediates may be performed as a pilot study for assurance of food safety.

\begin{tabular}{|c|}
\hline $\begin{array}{l}\text { Yuichi Nakajima }{ }^{1} \text {, Yuya Tanaka }{ }^{1}, \text { Kosuke Matsui }^{2} \text {, } \\
\text { Kazuyuki Maeda }^{1}, 3 \text {, Yoshiyuki Kitou }{ }^{1} \text {, } \\
\text { Kyoko Kanamaru }^{1} \text {, Shuichi Ohsato } \\
\text { Tetsuo Kobayashi }^{1} \text {, Naoko Takahashi-Ando }{ }^{2} \text {, } \\
\text { Makoto Kimura }^{1}\end{array}$ \\
\hline $\begin{array}{l}{ }^{1} \text { Department of Biological Mechanisms and } \\
\text { Functions, Graduate School of Bioagricultural } \\
\text { Sciences, Nagoya University, Furo-cho, Chikusa-ku, } \\
\text { Nagoya, Aichi 464-8601, Japan }\end{array}$ \\
\hline $\begin{array}{l}{ }^{2} \text { Graduate School of Science and Engineering, Toyo } \\
\text { University, Kujirai 2100, Kawagoe, Saitama 350-0815, } \\
\text { Japan }\end{array}$ \\
\hline $\begin{array}{l}{ }^{3} \text { Graduate School of Agriculture, Meiji University, } \\
\text { 1-1-1 Higashi-Mita, Tama-ku, Kawasaki, Kanagawa } \\
\text { 214-8571, Japan }\end{array}$ \\
\hline Correspondence \\
\hline $\begin{array}{l}\text { Yuichi Nakajima } \\
\text { E-mail: nakajima.yuichi@a.mbox.nagoya-u.ac.jp }\end{array}$ \\
\hline $\begin{array}{l}\text { (Received February 05, 2017, revised February 08, } \\
\text { 2017, accepted February 08, 2017) }\end{array}$ \\
\hline
\end{tabular}

\section{Acknowledgements}

This work was supported by a grant from the Tojuro Iijima Foundation for Food Science and Technology (grant number 27-19).

\section{Supplementary Materials}

Supplementary materials may be found in the online version of this article:

Supplementary Fig. S1

Supplementary Table 1

\section{References}

1) Kimura, M., Tokai, T., Takahashi-Ando, N., Ohsato, S., Fujimura, M.: Molecular and genetic studies of Fusarium trichothecene biosynthesis: pathways, genes, and evolution. Biosci Biotechnol Biochem, 71, 2105-2123 (2007)

2) Yoshizawa, T.: Thirty-five years of research on deoxynivalenol, a trichothecene mycotoxin: with special reference to its discovery and co-occurrence with nivalenol in Japan. Food Safety, 1, doi: 10.14252/foodsafetyfscj.2013002 (2013)

3) Maeda, K., Tanaka, A., Sugiura, R., Koshino, H., Tokai, T., Sato, M., Nakajima, Y., Tanahashi, Y., Kanamaru, K., Kobayashi, T., Nishiuchi, T., Fujimura, M., Takahashi-Ando, N., Kimura, M.: Hydroxylations of trichothecene rings in the biosynthesis of Fusarium trichothecenes: evolution of alternative pathways in the nivalenol chemotype. Environ Microbiol, 18, 3798-3811 (2016)

4) Nakajima, Y., Tokai, T., Maeda, K., Tanaka, A., TakahashiAndo, N., Kanamaru, K., Kobayashi, T., Kimura, M.: A set of heterologous promoters useful for investigating gene functions in Fusarium graminearum. JSM Mycotoxins, 64, 147-152 (2014)

5) Takahashi-Ando, N., Tokai, T., Yoshida, M., Fujimura, M., Kimura, M.: An easy method to identify 8-keto-15hydroxytrichothecenes by thin-layer chromatography. Mycotoxins, 58, 115-117 (2008)

6) Tokai, T., Koshino, H., Kawasaki, T., Igawa, T., Suzuki, Y., Sato, M., Fujimura, M., Eizuka, T., Watanabe, H., Kitahara, T., Ohta, K., Shibata, T., Kudo, T., Inoue, H., Yamaguchi, I., Kimura, M.: Screening of putative oxygenase genes in the Fusarium graminearum genome sequence database for their role in trichothecene biosynthesis. FEMS Microbiol Lett, 251, 193-201 (2005)

7) Kimura, M., Kaneko, I., Komiyama, M., Takatsuki, A., Koshino, H., Yoneyama, K., Yamaguchi, I.: Trichothecene 3$\mathrm{O}$-acetyltransferase protects both the producing organism and transformed yeast from related mycotoxins. Cloning and characterization of Tri101. J Biol Chem, 273, 1654-1661 (1998)

8) McCormick, S. P., Hohn, T. M., Desjardins, A. E.: Isolation and characterization of Tri3, a gene encoding 15-O-acetyltransferase from Fusarium sporotrichioides. Appl Environ Microbiol, 62, 353-359 (1996)

9) McCormick, S. P., Harris, L. J., Alexander, N. J., Ouellet, T., Saparno, A., Allard, S., Desjardins, A. E.: Tri1 in Fusarium graminearum encodes a P450 oxygenase. Appl Environ Microbiol, 70, 2044-2051 (2004)

10) Kadota, T., Kimura, M., Hirano, S., Tajima, O., Nakajima, T., Kamata, Y., Sugita-Konishi, Y.: Development of a simultaneous liquid chromatography/tandem mass spectrometric method for the determination of type B trichothecenes, their derivatives, and precursors in wheat. Rapid Commun Mass Spectrom, 25, 3481-3490 (2011)

11) Tokai, T., Takahashi-Ando, N., Izawa, M., Kamakura, T., Yoshida, M., Fujimura, M., Kimura, M.: 4-O-acetylation and 3-O-acetylation of trichothecenes by trichothecene 15-O-acetyltransferase encoded by Fusarium Tri3. Biosci Biotechnol Biochem, 72, 2485-2489 (2008)

12) Nakajima, Y., Kawamura, T., Maeda, K., Ichikawa, H., Motoyama, T., Kondoh, Y., Saito, T., Kobayashi, T., Yoshida, M., Osada, H., Kimura, M.: Identification and characterization of an inhibitor of trichothecene 3-O-acetyltransferase, TRI101, by the chemical array approach. Biosci Biotechnol Biochem, 77, 1958-1960 (2013) 
3-deCAL: $\left[\mathrm{C}_{17} \mathrm{H}_{24} \mathrm{O}_{5}+\mathrm{H}\right]^{+}$

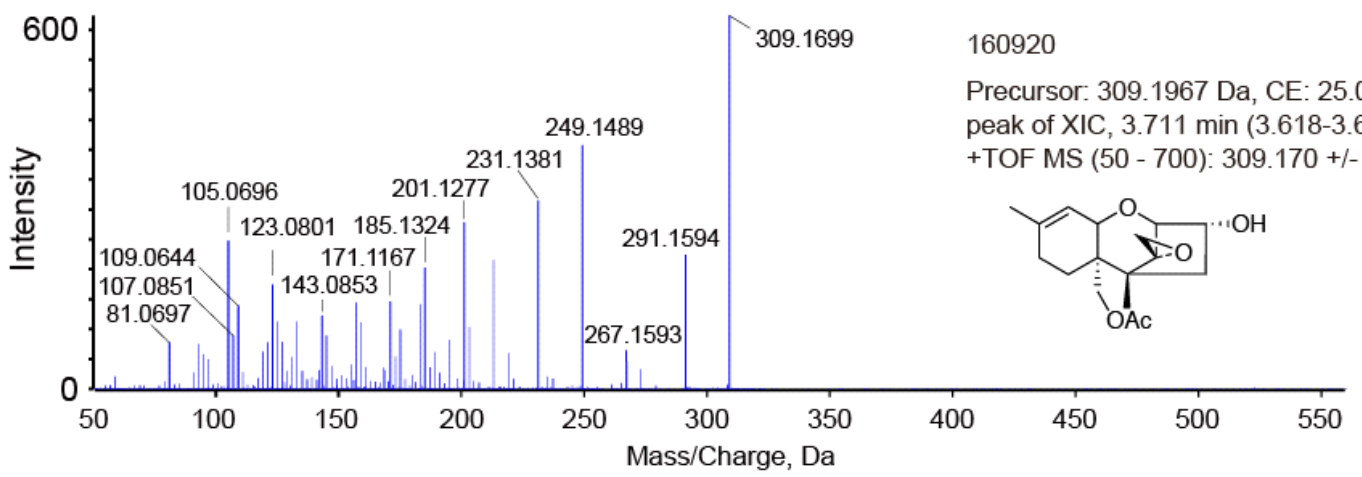

3-deCAL: $\left[\mathrm{C}_{17} \mathrm{H}_{24} \mathrm{O}_{5}+\mathrm{NH}_{4}\right]^{+}$

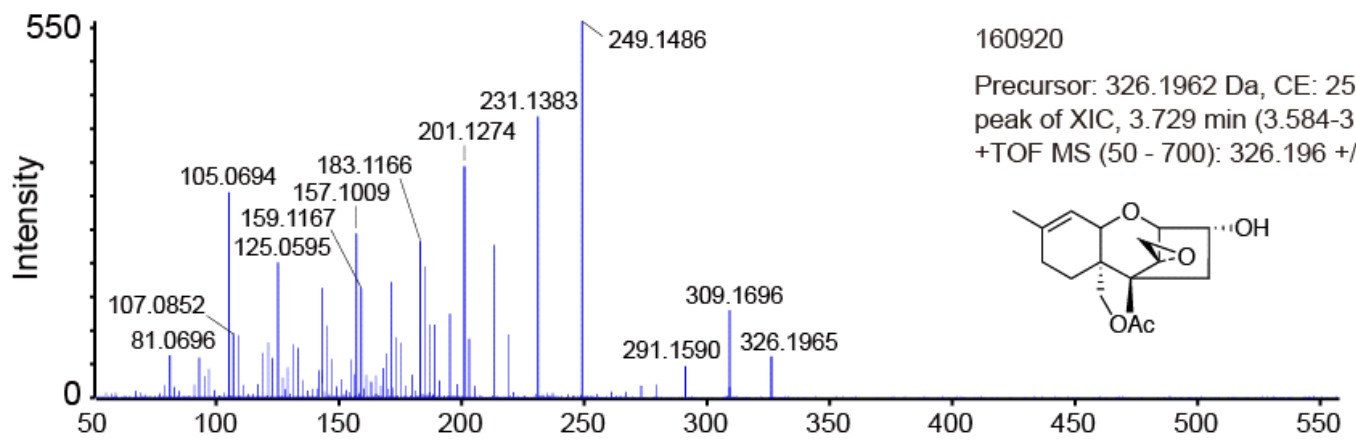

Supplementary Fig. S1 LC-MS/MS analyses of 3-deCAL standard. The tandem mass spectra of precursor ions detected in the positive ion mode are shown. 
Supplementary Table 1 Composition of the Met medium used in this study.

Methionine synthetic medium (Met medium) for submerged culture

\begin{tabular}{ll}
\hline element & concentration \\
\hline sucrose & $30 \mathrm{~g} / \mathrm{L}$ \\
$\mathrm{KH}_{2} \mathrm{PO}_{4}$ & $1 \mathrm{~g} / \mathrm{L}$ \\
$\mathrm{KCl}$ & $0.5 \mathrm{~g} / \mathrm{L}$ \\
$\mathrm{MgSO}_{4} \cdot 7 \mathrm{H}_{2} \mathrm{O}$ & $0.5 \mathrm{~g} / \mathrm{L}$ \\
trace elements $^{1}$ & $0.2 \mathrm{~mL} / \mathrm{L}$ \\
FeSO $_{4} \cdot 7 \mathrm{H}_{2} \mathrm{O}$ & $10 \mathrm{mg} / \mathrm{L}$ \\
methionine $^{1}$ & $5 \mathrm{mM}$ \\
\hline
\end{tabular}

1 filter-sterilized.

$5,000 \times$ Trace elements

\begin{tabular}{ll}
\hline element & concentration \\
\hline citric acid & $5 \mathrm{~g} / 100 \mathrm{~mL}$ \\
$\mathrm{MnSO}_{4} \cdot \mathrm{H}_{2} \mathrm{O}$ & $50 \mathrm{mg} / 100 \mathrm{~mL}$ \\
$\mathrm{ZnSO}_{4} \cdot 7 \mathrm{H}_{2} \mathrm{O}$ & $5 \mathrm{~g} / 100 \mathrm{~mL}$ \\
$\mathrm{H}_{3} \mathrm{BO}_{3}$ & $50 \mathrm{mg} / 100 \mathrm{~mL}$ \\
$\mathrm{Na}_{2} \mathrm{MoO}_{4} \cdot 2 \mathrm{H}_{2} \mathrm{O}$ & $50 \mathrm{mg} / 100 \mathrm{~mL}$ \\
$\mathrm{CuSO}_{4} \cdot 5 \mathrm{H}_{2} \mathrm{O}$ & $250 \mathrm{mg} / 100 \mathrm{~mL}$ \\
\hline
\end{tabular}

YG medium (for pre-culture)

\begin{tabular}{ll}
\hline element & concentration \\
\hline yeast extract & $5.0 \mathrm{~g} / \mathrm{L}$ \\
glucose & $20 \mathrm{~g} / \mathrm{L}$ \\
\hline
\end{tabular}

